\title{
An Indian Evidence-Based Study of Prevalence, Phenotypic Features, Lifestyle Modifications of Polycystic Ovarian Syndrome Patients
}

\author{
Roya Rozati ${ }^{1 *}$, Mohammad Akbar Ali², Wajeeda Tabasum ${ }^{3}$, Avinash Bardia ${ }^{4}$, Humaira Minhaj ${ }^{3}$, Avvari Bhaskara \\ Balaji ${ }^{5}$, Ayapati Mehdi Gautam ${ }^{3}$, Vikram Aiman Ayapati ${ }^{3}$, Taalia Nazeer Ahmed ${ }^{3}$ and Mohammed Sarosh Ahmed ${ }^{6}$ \\ ${ }^{1}$ Department of Obst \& Gynaecology, Shadan Institute of Medical Sciences and Maternal Health and Research Trust (MHRT), India \\ ${ }^{2}$ Department of General medicine, Bhaskara General Hospital and Medical College, India \\ ${ }^{3}$ Research Scholar, Maternal Health and Research Trust(MHRT), India \\ ${ }^{4}$ Scientist, Centre for Liver Research \& Diagnostics, India \\ ${ }^{5}$ Lab incharge ,IVF, Maternal Health and Research Trust (MHRT), India \\ ${ }^{6}$ Department of Community Medicine, Deccan College of Medical Sciences, India \\ Submission: May 30, 2021; Published: June 07, 2021 \\ *Corresponding author: Roya Rozati, Department of Obst \& Gynaecology, Shadan Institute of Medical Sciences and Maternal Health and Research \\ Trust (MHRT), India
}

\begin{abstract}
Objective: An Indian evidence-based study of Prevalence, phenotypic features, Lifestyle modifications of polycystic ovarian syndrome patients

Design: A large-scale population-based study was conducted among women of reproductive age in the state of Telangana between December 2018 and December 2020.

Setting: A randomized multi - stage stratified sampling method was adopted. PCOS screening was based on questionnaires. Blood samples for hormonal analysis were collected from those with probable and definitive PCOS cases.

Main Outcome Measure: Through our study we found the prevalence of polycystic ovarian syndrome in the Urban and rural areas of Hyderabad ,Telangana (South India) to be 6.5- 6.8\%

Results: In total, 688 patients were screened for PCOS,573 patients were enrolled of which 281 were from urban and 292 were from rural respectively. Of which probable PCOS cases 28 were probable PCOS and 17 were known cases and 83 were controls in the urban region.17 were probable PCOS and 10 were known cases and 73 were controls in the rural region. The overall count of probable cases were 45, Known PCOS were 27 and controls were 153.0f which 348 patients were not included due to various reasons were not included. Probable cases were 27.29 + 7.14,Controls cases were $29.67+6.88$ with ap value 0.001 had PCOS from Urban and Rural Area. The PCOS phenotypes were $8 \%$ clinical HA (hirsutism, H), alopecia (7\%), Acanthosis (3\%) Acne was (6\%), USG (35\%) in urban and Rural regions.55 \% and 45\% biochemical assessment done in Urban and Rural area respectively. Hormonal Assay was 75\% and $25 \%$ of the women with PCOS resided in Urban and Rural regions respectively. Hyperinsulinemia was observed in probable cases compared to the controls.
\end{abstract}

Keywords: PCOS; Infertility; Urban Region; Rural Region; NIH Criteria; LSM

Abbreviations: PCOS: Polycystic Ovarian Syndrome; LSM: Lifestyle Modifications: NIH: National Institute of Health

\section{Introduction}

Polycystic ovary syndrome (PCOS) is one of the most common disorder, yet most neglected, endocrinologic conditions affecting females of reproductive age of about $18-44$ and $6-20 \%$ [1]. is the prevalence worldwide. The condition is characterized by hyperandrogenism, ovulatory dysfunction and polycystic ovarian morphology (PCOM) - with excessive androgen production by the ovaries being a key feature of PCOS. The persistent hormonal 
disbalance leads to the complexities such as numerous cysts, an irregular menstrual cycle that ultimately leads to infertility among females. Stress, obesity, fluctuation in hormonal level is the major cause worldwide.

According to 2003 Rotterdam criteria now endorsed by the $\mathrm{NIH}$ and accepted internationally, the prevalence of PCOS ranges from $5.5 \%$ to $19.9 \%$ [2]. The syndrome is characterized with multiple gynecologic, dermatologic, metabolic, and psychologic aberrations like such as depression, anxiety, sexual dysfunction, and social problems, which affect a woman's identity and healthrelated quality of life (QOL) [3,4], of which hyperandrogenism, anovulation, and polycystic ovaries are the hallmark features [5], although not all patients have all three of these features. Polycystic ovary syndrome is one of the leading causes for infertility and is characterized by menstrual irregularities, hirsutism, cystic acne, seborrhea, hair loss, and obesity [6].

The increasingly high incidence of PCOS can be attributed to genetic factors, environmental factors, and intermarriages [7]; however, it is considered an amalgamation of insulin resistance, hyperandrogenemia, and factors causing follicular abnormalities [8]. Metabolic dysfunction characterized by insulin resistance and compensatory hyperinsulinemia is evident in the vast majority of affected individuals. PCOS has been strongly associated with the future development of type 2 diabetes mellitus, glucose intolerance, hyperinsulinemia, cardiovascular disorders, and hypertension $[9,10]$. Insulin resistance has been reported in $>60 \%$ of patients with PCOS [11], including obese and non-obese populations, and $10 \%$ of women with PCOS may develop type 2 diabetes by the age of 40 years. Elevated luteinizing hormone levels have been found to be significantly associated with PCOS related ovulation disorders and elevated anti-Mullerian hormone (AMH) levels in patients with PCOS [12].

In addition, its symptomatology shows significant heterogeneity across the lifecycle of women and affects several body organs. Diagnostic practices remain controversial and vary widely, primarily because of a lack of consensus criteria. Therefore, establishing a diagnosis of PCOS for adolescents and menopausal women is problematic. Currently, there are three sets of diagnostic criteria to define PCOS: NIH criteria 1990; Rotterdam criteria 2003; and Androgen Excess Society criteria 2006 [2]. The NIH criteria include only oligo an ovulation and hyperandrogenism [13]; the Rotterdam criteria define PCOS by the presence of a minimum of two features out of three (oligo an ovulation, polycystic ovaries, and clinical or biochemical hyperandrogenism); and hyperandrogenism is central to diagnosis of PCOS by the Androgen Excess Society criteria. Worldwide, PCOS is rapidly assuming epidemic proportions. In India, epidemiologic data regarding its prevalence and its complications are limited [14-17]. Furthermore, most reports are region -specific (mainly urban areas) and ignore urban/rural Accepted Article This article is protected by copyright. All rights reserved. comparisons in terms of prevalence [18-20]. It is likely that the prevalence of PCOS varies significantly between urban and rural regions owing to different environments, lifestyles, incidence of obesity, and dietary practices. The implementation of lifestyle -related modifications and screening of women with PCOS for the presence of associated risk factors is essential for planning substantial preventive health strategies. To date, however, there has been no nationally representative study on the prevalence of PCOS in the Hyderabad region of Southern India. Thus, the aim of the present study was to determine the prevalence of PCOS affecting the women of this region, and to assess the prevalence of depression, hypertension, and obesity among affected women, and their awareness of PCOS.

PCOS as a lifestyle disorder associated with modernization of living, they fail to include and compare population that follows modern and traditional lifestyle. Until now, three studies which report the prevalence of urban and rural Indian population were based in a hospital setting and thus biasing the population selection shown in (Figure 1).

Moreover, apart from the lack of disease awareness and management as well as medical therapy according to the guidelines, women, mostly in rural areas, are reluctant to visit gynecologists or endocrinologists for treatment despite having symptoms of the disease. This reluctance results in most patients remaining untreated, leading to various future complications. Therefore, the purpose of this study was to investigate the clinical aspects and QOL related to PCOS. Thus, the aim of the present study was to determine the prevalence of PCOS affecting the women of this region, and to assess the prevalence of depression, hypertension, and obesity among affected women, and their awareness of PCOS and vitamin D.

The objective of the present study was to determine the prevalence of PCOS in a well-defined population of unselected reproductive-aged women in the Urban and rural area of Hyderabad, India, aiming to evaluate a large number of subjects and perform a complete phenotyping of the women involved.

\section{Materials and Methods}

Ethical approval Ethical approval was obtained from the Ethics Review Board of each participating hospital namely institutional review board for bioethics, at MHRT hospital, Hyderabad. Informed consent was obtained from each patient after explaining the research and its objectives. Patients were included only after they signed the informed consent. All researchers ensured patient data confidentiality and compliance with the Declaration.

\section{Study Setting and Design}

In this prospective, cross-sectional, observational study, patients with PCOS were included who were screened at hospital or who visited their outpatient departments for a routine checkup Maternal health research trust hospital at Hyderabad. 


\section{Study Sample}

The study sample comprised females of reproductive age who met the inclusion criteria based on the NIH 1990 criteria for PCOS and were admitted to or visited the outpatient department of any of the participating hospital -Maternal health research trust hospital at Hyderabad for a routine checkup as well as those who had infertility problems or repeated miscarriages and visited infertility clinics from May 2018 to May 2020. It is a populationbased study conducted in the state of Telangana, Which included 8 urban constituencies and 10 rural constituencies of the state. The study was carried out from May 2018-May 2020. The diagnostic criteria of PCOS on the basis of the NIH 1990 criteria [14-17] include hyperandrogenism and oligo ovulation and exclude other disorders mimicking PCOS, such as adult-onset congenital adrenal hyperplasia, hyperprolactinemia, and androgen-secreting neoplasms. Inclusion criteria Females were included in the study based on the following inclusion criteria: (a) reproductive age (1840 years), (b) history of infertility or repeated miscarriage, and (c) diagnosis of PCOS by the consulting gynecologist/physician based on the NIH 1990 criteria(Figure 1: Flowchart of the study population).

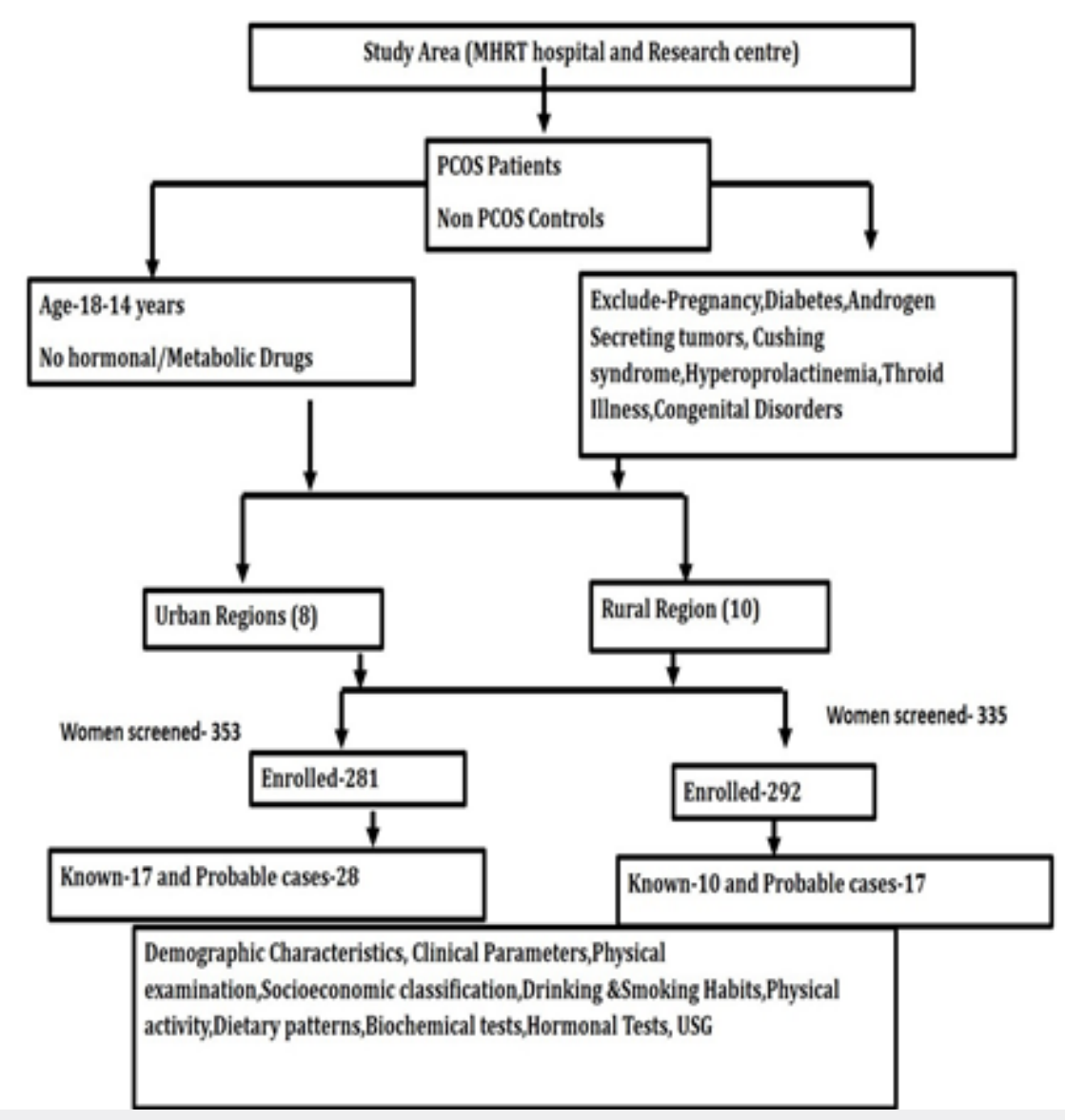

Figure 1: depicts the flowchart of the study Population which is conducted in the state of Telangana which constitutes 8 urban constituencies and 10 rural constituencies, and the study was carried out from May 2018 to May 2020.Screening of Women was done in total of 353 and 335 Women including the urban and rural areas of Telangana, respectively.

A stage- wise procedure was followed for conducting the study systematically. In the first phase, we surveyed the community using questionnaires and in the next phase, we confirmed the cases of PCOS. Later Assays were performed followed by statistical analysis of data and obtaining the results. This is a two- stage, cross- sectional, community-based study. The study population included community- dwelling women aged 15-40 years. They were recruited from both rural and urban areas which were located near our institution based on logistics and convenience. The sampling strategy involved selecting an urban ward or rural block based on proximity. The electoral roll was procured from 3-4 polling booths for both rural and urban. Informed consent had been obtained from each participant after a detailed explanation of the study. The screening was done using pre-designed simple screening questionnaires at all the constituencies which are based on few symptoms about the main features of PCOS including 
menstrual history, self hirsutism score, weight gain, acne, and some important risk factors which were determined by trained healthcare and answers to these questionnaires were re-confirmed after an oral interview to screen the probable PCOS.

The questionnaire has 21 questions, which have three domains: A. Personal information; B. Anthropometry; C. PCOS related questions. The domain $\mathrm{C}$ included 13 questions focused on features of hyperandrogenism, mensuration, and insulin resistance. The women who had at least one feature of hyperandrogenism or oligomenorrhea with or without obesity and insulin resistance were taken as probable cases of PCOS on the screening questionnaire. Such women who were probable cases were invited to our institute for a detailed clinical, biochemical and hormonal assessment of the follicular phase of their cycle (2nd -7th day). About $20 \%$ of women who tested negative on the questionnaire were also invited for a detailed work-up identical to that of women who were positive for the questionnaire. This will also serve to validate the usefulness of this particular questionnaire (in part or total) for future epidemiological surveys of PCOS. Occupation, diet, attitude, knowledge regarding PCOS, and daily lifestyle of such subjects who were probable were noticed. The participants were asked for symptoms relating to hirsutism, acne vulgaris, menstrual cycle irregularities, loss of hair, or any other chronic illness. A brief examination of a total of nine body parts was carried out which included the upper lip, upper and lower abdomen, chest, chin, upper and lower back, thighs, upper arms were examined for the presence of terminal hair and were graded from $0-4$.

The Ferriman- Gallwey score was assessed as the cumulative score of all these sites. Height, weight, hip and waist circumference, and blood pressure were the anthropometric measurements in the study. Subjects without menstrual irregularities were assumed not to have PCOS and were hence labeled as control subjects. Besides in the community pool, the women who had a known diagnosis of PCOS with documentation of USG and hormones were recorded as positive and were also invited for future investigations if drugnaive.

All the women who are drug- naive had undergone clinical assessment (anthropometry, hirsutism, acne, oligomenorrhea, androgenic alopecia, primary infertility), biochemical evaluation (blood biochemistry, OGTT), and hormonal estimations (T4, TSH, PRL, LH, FSH, total testosterone).

\section{Exclusion Criteria}

Females were excluded from the study if they met either of the following conditions: (a) age of 45 years and (b) no PCOS diagnosis by the consulting gynecologist/physician. Initially, all patients were diagnosed based on the NIH 1990 criteria as per local practices. The researchers further investigated each patient based on the Rotterdam criteria as well as conducted detailed investigation of menstrual history to identify irregular or absent ovulation and ultrasonography to identify ovarian morphology.

\section{Sample Size}

The study sample size was determined using the Epi Info Stat Calc software [21,22] and a confidence level of 95\%. Accordingly, an approximate sample size of 165 was considered statistically significant. After considering $20 \%$ of non-response or missing data values, the final required sample size was determined to be 198.

\section{Sampling Technique}

The present study used convenience sampling for patient selection.

\section{Statistical Analysis}

All data collected using the clinical checklist and SF-12 questionnaire were recorded in an SPSS spreadsheet after coding and carefully defining all the studied variables. Analysis was then performed in two steps. The first step included descriptive analysis wherein patients' clinical condition and various variables were analyzed for the respective frequencies, percentages, and measure of central tendency. In the second step, inferential statistics were used to determine the associations among patient- and therapyrelated variables, clinical outcomes, and QOL using Pearson's chisquare test, analysis of variance (ANOVA), and post hoc analysis, with a p-value of $<0.05$ indicating statistical difference.

\section{Result}

In total, 688 patients were screened for PCOS, 573 patients were enrolled of which 281 were from urban and 292 were from rural, respectively. Of which probable PCOS cases 28 were probable PCOS and 17 were known cases and 83 were controls in the urban region.17 were probable PCOS and 10 were known cases and 73 were controls in the rural region. The overall count of probable cases were 45, Known PCOS were 27 and controls were 153.Of which 348 patients were not included due to various reasons were not included. Among these, 153 were of mean aged 29 years and 45 mean aged 27 years with a p value of 0.045 . Bar graph showing mean QOL scores according to clinical conditions in patients with PCOS in comparison with Controls. Among total 688 women screened, Probable cases were 27.29+7.14,Controls cases were 29.67+6.88 with ap value 0.001 had PCOS from Urban and Rural Area. The PCOS phenotypes were $8 \%$ clinical HA (hirsutism, H), alopecia (7\%), Acanthosis (3\%) Acne was (6\%), USG (35\%) in urban and Rural regions.55 \% and 45\% biochemical assessment done in Urban and Rural area respectively. Hormonal Assay was $75 \%$ and $25 \%$ of the women with PCOS resided in Urban and Rural regions respectively. PCOS increases the risk for type 2 diabetes mellitus, gestational diabetes and other pregnancy-related complications, venous thromboembolism, cerebrovascular and cardiovascular events and endometrial cancer. PCOS is a diagnosis of exclusion, based primarily on the presence of hyperandrogenism, ovulatory dysfunction and PCOM. 
The 573women were classified as PCOS or non-PCOS on the basis of three major diagnostic criteria: NIH 1990; Rotterdam 2003; and AEPCOS 2006.Of which probable PCOS cases were 45, controls were 153 by NIH criteria Anthropometric measurements and laboratory investigations of study participants are summarized in Table 1. Women in the definite PCOS group had significantly fewer number of cycles per year, higher Ferriman-Gallwey score, lowdensity lipoprotein, testosterone. 1-hour plasma glucose levels post 75-g glucose and lower levels of high-density lipoprotein and follicle-stimulating hormone compared to controls. There was no significant difference in the age, no of cycles previous year or FG score but there was a significant difference in body mass index (BMI, calculated as weight in kilograms divided by the square of height in meter) when compared to urban and rural population in Figures $2 \& 3$.

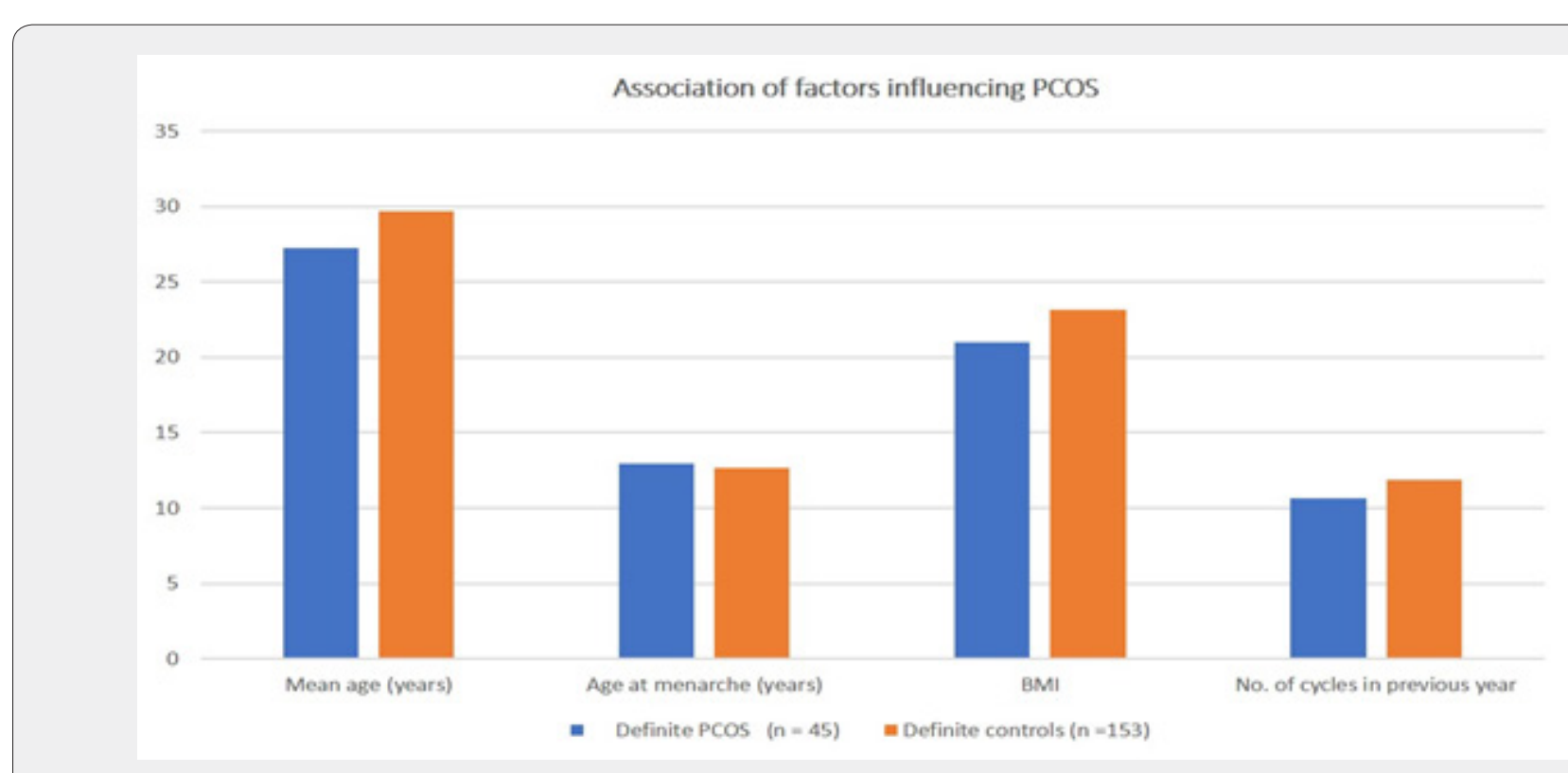

Figure 2: depicts the Association of factors influencing PCOS.

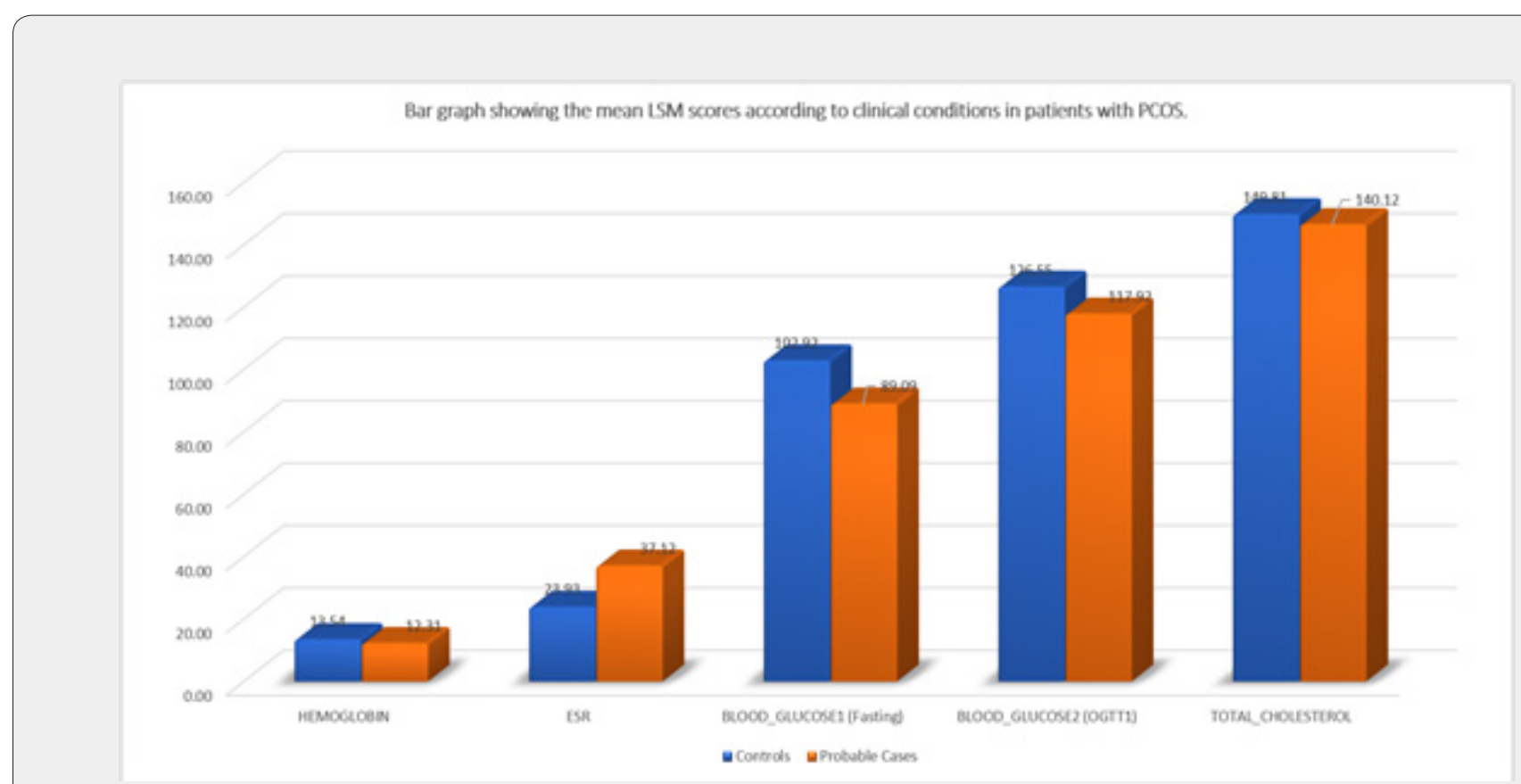

Figure 3: depicts the mean lifestyle modifications scores according to clinical conditions in patients with PCOS in comparison with controls. 
Table 1: shows that 198 patient's which were included of which probable PCOS cases were 45, controls were 153 patients which included all the parameters in the study such as Physical Examination, biochemical parameters and Hormonal assay.

\begin{tabular}{|c|c|c|c|}
\hline Parameters & $\begin{array}{l}\text { Definite PCOS } \\
\quad(\mathrm{N}=45)\end{array}$ & Definite Controls ( $N=153$ ) & P Value \\
\hline Mean age (years) & $27.28 \pm 7.14$ & $29.66 \pm 6.87$ & 0.045 \\
\hline Age at menarche (years) & $12.97 \pm 1.13$ & $12.66 \pm 0.74$ & 0.08 \\
\hline Menstrual cycle irregularity & $0.57 \pm 0.49$ & $0.06 \pm 0.24$ & 0.001 \\
\hline No. of cycles in previous year & $10.68 \pm 3.06$ & $11.89 \pm 0.65$ & 0.01 \\
\hline Longest cycle & $38.24 \pm 18.53$ & $30.49 \pm 3.62$ & 0.007 \\
\hline Shortest cycle & $27.33 \pm 3.78$ & $27.27 \pm 1.72$ & 0.91 \\
\hline Weight & $52.07 \pm 12.71$ & $55.71 \pm 12.93$ & 0.09 \\
\hline Height & $148.47 \pm 7.17$ & $149.95 \pm 10.93$ & 0.39 \\
\hline Tricep thickness & $38.22 \pm 13.59$ & $32.44 \pm 12.83$ & 0.009 \\
\hline Dorsum thickness & $48.44 \pm 9.71$ & $45.46 \pm 10.64$ & 0.09 \\
\hline Blood pressure 1 & $111.40 \pm 15.147$ & $114.81 \pm 10.87$ & 0.16 \\
\hline Blood pressure 2 & $73.29 \pm 9.89$ & $77.30 \pm 8.59$ & 0.008 \\
\hline Hemoglobin & $12.31 \pm 1.99$ & $13.54 \pm 13.29$ & 0.53 \\
\hline ESR & $37.12 \pm 22.56$ & $23.93 \pm 16.27$ & 0.0001 \\
\hline BG- fasting & $89.09 \pm 10.49$ & $102.92 \pm 60.42$ & 0.12 \\
\hline BG- OGTT 1 & $117.92 \pm 34.48$ & $126.55 \pm 66.77$ & 0.6 \\
\hline BG -OGTT 2 & $104.76 \pm 12.92$ & $123.71 \pm 62.37$ & 0.13 \\
\hline TSH & $3.61 \pm 4.54$ & $2.81 \pm 1.82$ & 0.25 \\
\hline $\mathrm{T} 4$ & $8,88 \pm 2.3$ & $8.29 \pm 2.39$ & 0.14 \\
\hline Prolactin & $28.64 \pm 60.54$ & $20.33 \pm 28.74$ & 0.38 \\
\hline Cortisol & $8.48 \pm 4.61$ & $8.86 \pm 5.13$ & 0.65 \\
\hline LH & $13.32 \pm 13.09$ & $13.88 \pm 14.50$ & 0.82 \\
\hline FSH & $10.47 \pm 16.04$ & $10.47 \pm 16.04$ & 1 \\
\hline Testosterone & $0.11 \pm 0.321$ & $0.11 \pm 0.315$ & 1 \\
\hline SHBG & $61.39 \pm 34.56$ & $67.34 \pm 35.91$ & 0.33 \\
\hline E2 & $4.95 \pm 31.32$ & $1.12 \pm 3.19$ & 0.42 \\
\hline C peptide & $2.95 \pm 2.06$ & $2.38 \pm 2.03$ & 0.1 \\
\hline Insulin 1 & $38.61 \pm 48.83$ & $19.82 \pm 20.08$ & 0.01 \\
\hline Insulin 2 & $81.02 \pm 66.73$ & $53.05 \pm 38.76$ & 0.01 \\
\hline Insulin 3 & $73.93 \pm 67.08$ & $50.66 \pm 37.57$ & 0.03 \\
\hline Bleeding (Heavy/Normal) & $2.57 \pm 0.78$ & $2.79 \pm 0.53$ & 0.08 \\
\hline Painful period & $0.31 \pm 0.46$ & $0.10 \pm 0.30$ & 0.005 \\
\hline Days of bleeding & $4.22 \pm 1.18$ & $4.33 \pm 0.92$ & 0.6 \\
\hline Mid cycle bleeding & $0.04 \pm 0.20$ & $0.03 \pm 0.17$ & 0.73 \\
\hline Presence of clots & $0.15 \pm 0.36$ & $0.06 \pm 0.24$ & 0.12 \\
\hline FG Score & $0.08 \pm 0.46$ & $0.01 \pm 0.11$ & 0.29 \\
\hline Facial acne & $0.08 \pm 0.28$ & $0.01 \pm 0.11$ & 0.1 \\
\hline Atypical acne & $0.44 \pm 0.20$ & $0.03 \pm 0.19$ & 0.75 \\
\hline Acanthosis & $0.11 \pm 0.31$ & $0.03 \pm 0.17$ & 0.1 \\
\hline Alopecia & $0.17 \pm 0.38$ & $0.00 \pm 0.00$ & 0.04 \\
\hline Family history & $0.00 \pm 0.00$ & $0.01 \pm 0.13$ & 0.52 \\
\hline Hirsutism & $0.02 \pm 0.14$ & $0.0065 \pm 0.08085$ & 0.52 \\
\hline
\end{tabular}


Journal of Gynecology and Women's Health

\begin{tabular}{|c|c|c|c|}
\hline Uterine width & $3.28 \pm 1.55$ & $0.20 \pm 11.75$ & 0.001 \\
\hline Uterine length & $6.06 \pm 1.88$ & $6.83 \pm 8.84$ & 0.58 \\
\hline Uterine thickness & $3.86 \pm 1.41$ & $5.54 \pm 6.28$ & 0.09 \\
\hline Endometrium thickness & $7.19 \pm 2.46$ & $6.86 \pm 2.61$ & 0.45 \\
\hline Fluid pelvis & $7.19 \pm 2.46$ & $0.07 \pm 0.27$ & 0.001 \\
\hline Right ovary follicle no. & $8.27 \pm 2.63$ & $6.09 \pm 2.69$ & 0.001 \\
\hline Left ovary follicle no. & $7.60 \pm 2.90$ & $6.30 \pm 3.01$ & 0.01 \\
\hline
\end{tabular}

The aggregation of CV risk factors in PCOS patients and their relative contributions of BMI and insulin levels remain controversial. Studies using obesity or insulin as univariate predictors of $\mathrm{CV}$ risk have found statistically significant relationships, but there are few studies which have examined the interaction directly. After correction for BMI, our data show significant relationships of insulin level with waist circumference and lipid levels but not with waist-to-hip ratio, fasting glucose level, or blood pressure. Table 2 shows that among the definite PCOS patients, CV risk factors are up to five times as prevalent as among the control patients have been discussed in Table 2 .

Table 2: Prevalence of risk factors in PCOS according to insulin level.

\begin{tabular}{|c|c|c|c|}
\hline Parameters & Definite PCOS (n= 45) & Definite Controls (n=153) & P Value \\
\hline Waist circumference & $70.33 \pm 20.75$ & $67.44 \pm 24.35$ & 0.47 \\
\hline BMI & $21.02 \pm 6.81$ & $23.12 \pm 7.66$ & 0.09 \\
\hline Cholesterol & $146.60 \pm 29.21$ & $149.81 \pm 39.60$ & 0.61 \\
\hline HDL & $44.96 \pm 11.17$ & $44.20 \pm 10.49$ & 0.67 \\
\hline LDL & $86.16 \pm 23.35$ & $92.12 \pm 28.48$ & 0.2 \\
\hline VLDL & $24.11 \pm 14.43$ & $24.50 \pm 13.89$ & 0.87 \\
\hline TG & $121.47 \pm 71.63$ & $124.16 \pm 70.66$ & 0.82 \\
\hline
\end{tabular}

In Figures 4 \& 5 describes Patients with PCOS have higher insulin levels than controls across the range of BMI (Figure 3), with probable PCOS and controls in have been shown to be insulin

resistant. This suggests that PCOS itself confers insulin resistance, with the added effect of obesity.

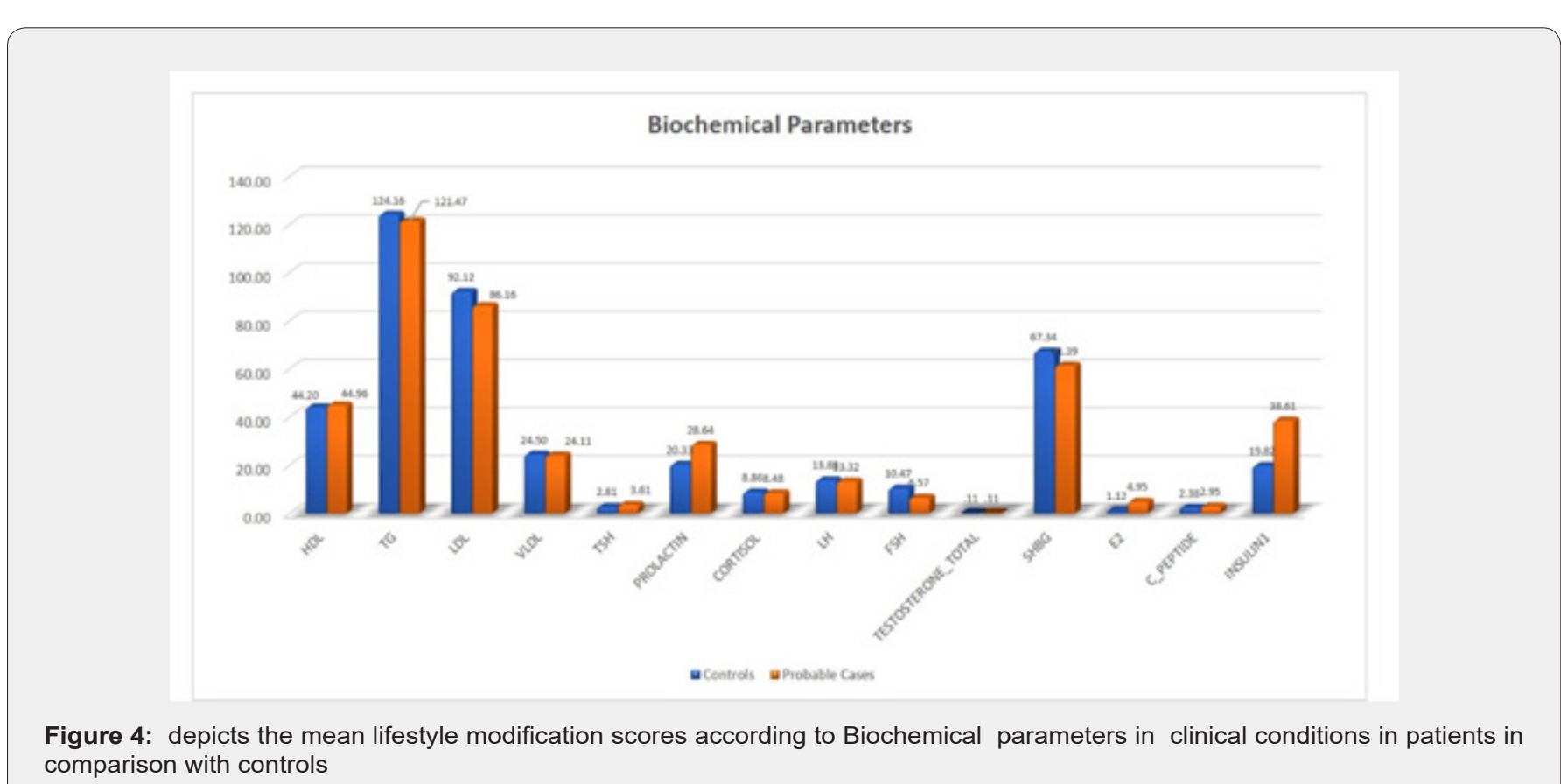


Journal of Gynecology and Women's Health

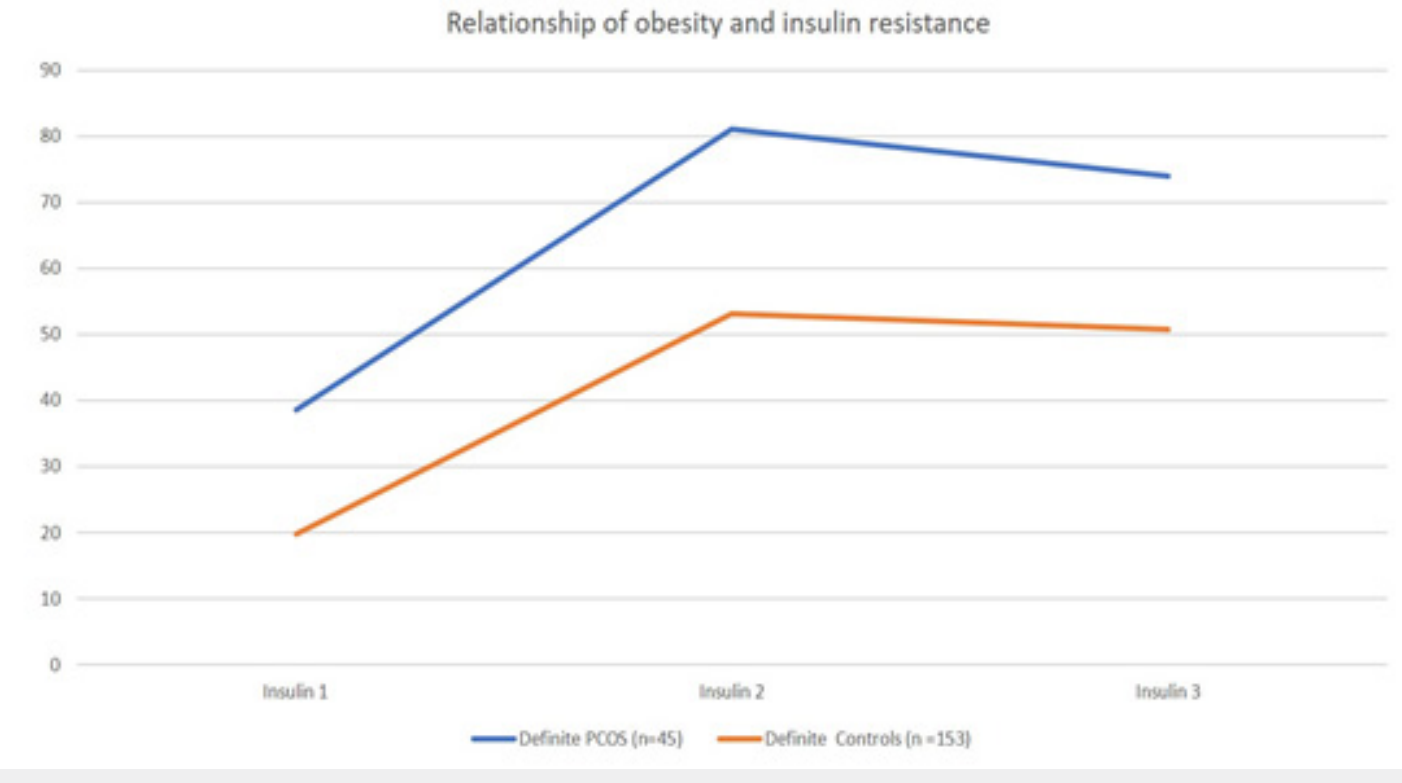

Figure 5: depicts relationship of obesity and insulin resistance in comparision with definite pcos (45) and Definite controls(153).

\section{Discussion}

In this prospective study is assessed under the NIH criteria to be prevalence of probable PCOS cases was $8.8 \%$ which is close to the study conducted in Central India which is $8.2 \%$ [23]. Our results are in closely agreement with study of Choudhary et al (9.13\%.)Vaidya et al [24] (3.4\% of women) found that globally, prevalence estimates of PCOS are highly variable, ranging from $2.2 \%$ to as high as $26 \%$.This value was slightly higher than the $6.5-6.8 \%$ obtained in three other prevalence studies that also used the NIH criteria. [25-28]. The methodological strengths of this study include it being the largest and only communitybased prevalence study of PCOS to be carried out on a Urban and Rural areas of Hyderabad in Southern India. Unfortunately, no statistical evidence was supplied in these prevalence studies to determine the representativeness of participants. Furthermore, the representation of prevalence under the current diagnostic criteria, as well as each of the criteria separately permits values obtained in this study to be compared with a wider range of other studies.

The study population is also comparable to other western populations [29] in terms of levels of obesity and waist circumference, although the levels reported are lower than those reported in the USA [30,31]. As a consequence, this is one of the most representative studies of PCOS prevalence in Caucasian women to date. Women in the broader age range of 15-40 years have been included in the present study, thus making the results more representative of the general population. This is the first study of PCOS in Hyderabad, with a population of mixed ethnic background and marked variation in dietary and cultural traits. From our study, we noted an interesting fact that the prevalence in rural population is less while compared to those from urban area. However, the reason for comparatively less PCOS cases may be due to lack of awareness and/or minimized or nil exposure to junk foods, pollution and other endocrine disruptors. Moreover, Women in rural areas do not depend on labour saving devices for household work or vehicles for transport thus helping them maintain a good BMI.

The ideal BMI is 18.5 to 24.9 range in women. These characteristics provide a unique opportunity to investigate the association of lifestyle, environmental, genetic and epigenetic factors with the phenotypic expression of PCOS. We hypothesize that the prevalence of metabolic and reproductive features in women with PCOS may vary in different regions of the country and in different PCOS phenotypes. Management of hyperandrogenism requires long-term and multi-dimensional treatment. This involves a combination of lifestyle modification, mechanical hair removal methods and pharmacological therapy for androgenic suppression.

Lifestyle modifications- Insulin resistance, hyperandrogenism, and the severity of PCOS can be improved through lifestyle modification (LSM), such as dietary modifications, physical exercises, or behavioral changes, medications, such as metformin, or bariatric surgery will improve the body composition, hyperandrogenism and IR in women with PCOS [32,33]. Weight loss has positive effects on the clinical improvement in menstrual function, fertility, pregnancy outcomes, and endocrine parameters 
[34]. These changes occurred with minimal weight loss, which is encouraging for women who have constantly failed to achieve reproductive success on a variety of short-term low-calorie diets. Changes in insulin sensitivity and falling serum insulin values may be the metabolic mediator of these results.

\section{Implications}

LSM programs should be recommended as first-line treatment and as a part of any management plan for women with PCOS, given the limited risk and overall likely, albeit modest, benefit with such an intervention. Indirect evidence regarding the benefits of LSM can also be derived from non-PCOS settings, such as individuals with type 2 diabetes [35,36] and obesity [37,38]. This is particularly important, considering the minimal harm and overall low cost of such interventions and the additional cardiovascular and metabolic benefits associated with LSM.

\section{Conclusion}

Based on the NIH diagnostic criteria, there is a similar prevalence of PCOS between $6 \%-10 \%$ is documented in the world. PCOS is a heterogeneous clinical syndrome with a multifaceted pathogenesis and is associated with lifelong morbidity. The clinical manifestations of PCOS include oligomenorrhoea, hirsutism, excessive acne and hair loss. The estimated prevalence in India ranged from 6-20\%. Considering the vast diversity in the population of India, large-scale community-based studies using internationally accepted criteria, in various geographic regions, are necessary to shed light on the actual prevalence of this disorder and more research should be done. The various metabolic, inflammatory and autoimmune components including the obesity-related cytokines and oxidative stress markers in relation to the pathogenesis of PCOS Therefore, PCOS management guidelines should review the recommendations regarding the use of pharmacological agents for these conditions. The clinical conditions and complications associated with PCOS should be given due importance while selecting appropriate management plans for each patient considering the effects of these conditions on overall morbidity and LSM. There are many hypothesized reasons for the lack of understanding of the risk and diagnosis of PCOS and one main reason could be the conflicting diagnostic standards. Management and treatment of PCOS included healthy diet, regular physical activity, and medications, which address the associated manifestations and co-morbidities. LSM tends to have more benefits such as improvement in the menstrual frequency and pregnancy outcomes and should be the primary recommendation in women with PCOS. Clinicians prescribing LSM interventions must consider the patient's capacity to sustain diet and exercise adherence and weight maintenance over time for the clinical benefits on PCOS to continue.

\section{Limitations of the Study}

The cross-sectional design of this study does not allow causal conclusions, and as such, the interpretability of our findings is limited. Only a limited number of patients turned up.

\section{Author Contributions}

Dr. Roya Rozati facilitated the initial conception of the idea to research the topic, participated in the drafting of manuscript, and read and approved the final manuscript. Dr. Roya Rozati researched the initial idea and converted the research to focus on prevalence, researched the existing literature, drafted the manuscript, and read and approved the final draft. Wajeeda Tabasum, Dr. Avinash Bardia researched the existing literature, participated in the drafting of the manuscript, and read and approved the final draft. Wajeeda Tabasum, Dr. Avinash Bardia and Humaira Minhaj researched the existing literature, participated in drafting the manuscript, and read and approved the final draft.

\section{Acknowledgment}

The authors thank ICMR for providing support for the duration of this project and for their assistance with manuscript preparation.

\section{Funding}

This study was funded by the ICMR

\section{Conflicts of Interest}

The authors declare that they have no conflict of interest.

\section{References}

1. Bozdag G, Mumusoglu S, Zengin D, Karabulut E, Yildiz BO, et al. (2016) The prevalence and phenotypic features of polycystic ovary syndrome: a systematic review and meta-analysis. Hum Reprod 31(12): 28412855.

2. Lizneva D, Suturina L, Walker W, Brakta S, Gavrilova-Jordan L, et al. (2016) Criteria, prevalence, and phenotypes of polycystic ovary syndrome. Fertil Steril 106(1): 6-15.

3. Hung JH, Hu LY, Tsai SJ, Yang AC, Huang MW, et al. (2014) Risk of psychiatric disorders following polycystic ovary syndrome: a nationwide population-based cohort study. PloS one 9(5): e97041.

4. Greenwood EA, Pasch LA, Cedars MI, Legro RS, Huddleston HG, et al. (2018) Association among depression, symptom experience, and quality of life in polycystic ovary syndrome. Am J Obstet Gynecol 219(3): 279-e1-279-e7.

5. Ndefo UA, Eaton A, Green MR (2013) Polycystic ovary syndrome: a review of treatment options with a focus on pharmacological approaches. P T 38(6): 336-355.

6. Khomami MB, Tehrani FR, Hashemi S, Farahmand M, Azizi F, et al. (2015) Of PCOS symptoms, hirsutism has the most significant impact on the quality of life of Iranian women. PLoS One 10(4): e0123608.

7. Brady C, Mousa SS, Mousa SA (2009) Polycystic ovary syndrome and its impact on women's quality of life: More than just an endocrine disorder. Drug Healthc Patient Saf 1: 9-15.

8. Dumesic DA, Akopians AL, Madrigal VK, Ramirez E, Margolis DJ, et al. (2016) Hyperandrogenism accompanies increased intra-abdominal fat storage in normal weight polycystic ovary syndrome women. J Clin Endocrinol Metab 101(11): 4178-4188. 
9. Franks S (1995) Polycystic ovary syndrome. N Engl J Med 333(13): 853-861.

10. Christakou CD, Diamanti-Kandarakis E (2008) Role of androgen excess on metabolic aberrations and cardiovascular risk in women with polycystic ovary syndrome. Womens Health (Lond) 4(6): 583-594.

11. Zhu Q, Zhou H, Zhang A, Gao R, Yang S, et al. (2016) Serum LBP is associated with insulin resistance in women with PCOS. PLoS One 11(1): e0145337.

12. Akram M, Roohi N (2015) Endocrine correlates of polycystic ovary syndrome in Pakistani women. J Coll Physicians Surg Pak 25(1): 22-26.

13. Roe AH, Dokras A (2011) The diagnosis of polycystic ovary syndrome in adolescents. Rev Obstet Gynecol 4(2): 45.

14. Ganie MA, Marwaha RK, Aggarwal R, Singh S (2010) High prevalence of polycystic ovary syndrome characteristics in girls with euthyroid chronic lymphocytic thyroiditis: a case-control study. Eur J Endocrinol 162(6): 1117-11122.

15. Nidhi R, Padmalatha V, Nagarathna R, Amritanshu R (2011) Prevalence of polycystic ovarian syndrome in Indian adolescents. J Pediatr Adolesc Gynecol 24(4): 223-227.

16. Vijayan CP, Sonia A (2013) Prevalence of Polycystic Ovary Syndrome among students of a teaching collegiate hospital.

17. Joshi B, Mukherjee S, Patil A, Purandare A, Chauhan S, et al. (2014) A cross-sectional study of polycystic ovarian syndrome among adolescent and young girls in Mumbai, India. Indian J Endocrinol Metab18(3): 317-324.

18. Joseph N, Reddy AG, Joy D, Patel V, Santhosh P, et al. (2016) Study on the proportion and determinants of polycystic ovarian syndrome among health sciences students in South India. J Nat Sci Biol Med 7(2): 166172.

19. Choudhary A, Jain S, Chaudhari P (2017) Prevalence and symptomatology of polycystic ovarian syndrome in Indian women: is there a rising incidence. International Journal of Reproduction, Contraception, Obstetrics and Gynecology 6(11): 4971-4975.

20. Gupta M, Singh D, Toppo M, Priya A, Sethia S, et al. (2018) A cross sectional study of polycystic ovarian syndrome among young women in Bhopal, Central India. Int J Community Med Public Health 5(1): 95100

21. Fosgate GT (2009) Practical sample size calculations for surveillance and diagnostic investigations. J Vet Diagn Invest 21(1): 3-14.

22. Bergström E, Blomquist HK (2009) Is the prevalence of overweight and obesity declining among 4-year-old Swedish children? Acta paediatrica 98(12):1956-1958.

23. Nidhi R, Padmalatha V, Nagarathna R, Amritanshu R (2011) Prevalence of polycystic ovarian syndrome in Indian adolescents. J Pediatr Adolesc Gynecol 24(4): 223-227.

24. Vaidya R, Joshi B (2016) PCOS-epidemic in India: An emerging public health challenge. International Conf PCOS Society India with AE-PCOS Society USA.
25. Diamanti-Kandarakis E, Kouli CR, Bergiele AT, Filandra FA, Tsianateli TC, et al. (1999) A survey of the polycystic ovary syndrome in the Greek island of Lesbos: hormonal and metabolic profile. J Clin Endocrinol Metab 84(11): 4006-4011.

26. Michelmore KF, Balen AH, Dunger DB, Vessey MP (2000) Polycystic ovaries and associated clinical and biochemical features in young women. Obstetrical \& Gynecological Survey 55(8): 494-496.

27. Asunción M, Calvo RM, Millán SJL, Sancho J, Avila S, et al. (2000) A prospective study of the prevalence of the polycystic ovary syndrome in unselected Caucasian women from Spain. J Clin Endocrinol Metab 85(7): 2434-2438.

28. Razak F, Anand S, Vuksan V, Davis B, Jacobs R, et al. (2005) Ethnic differences in the relationships between obesity and glucose-metabolic abnormalities: a cross-sectional population-based study. Int J Obes (Lond) 29(6): 656-667.

29. Flegal KM, Carroll MD, Ogden CL, Johnson CL (2002) Prevalence and trends in obesity among US adults, 1999-2000. JAMA 288(14): 17231727.

30. Zhu S, Wang Z, Heshka S, Heo M, Faith MS, et al. (2002) Waist circumference and obesity-associated risk factors among whites in the third National Health and Nutrition Examination Survey: clinical action thresholds. Am J Clin Nutr 76(4): 743-749.

31. Jensterle M, Kocjan T, Kravos NA, Pfeifer M, Janez A, et al. (2015) Shortterm intervention with liraglutide improved eating behavior in obese women with polycystic ovary syndrome. Endocr Res 40(3): 133-138.

32. Vosnakis C, Georgopoulos NA, Rousso D, Mavromatidis G, Katsikis I, et al. (2013) Diet, physical exercise and Orlistat administration increase serum anti-Müllerian hormone (AMH) levels in women with polycystic ovary syndrome (PCOS). Gynecol Endocrinol 29(3): 242-245.

33. Clark AM, Ledger W, Galletly C, Tomlinson L, Blaney F, et al. (1995) Weight loss results in significant improvement in pregnancy and ovulation rates in anovulatory obese women. Hum Reprod 10(10): 2705-2712.

34. Yamaoka K, Tango T (2005) Efficacy of lifestyle education to prevent type 2 diabetes: a meta-analysis of randomized controlled trials. Diabetes care 28(11): 2780-2786.

35. Esposito K, Kastorini CM, Panagiotakos DB, Giugliano D (2010) Prevention of type 2 diabetes by dietary patterns: a systematic review of prospective studies and meta-analysis. Metab Syndr Relat Disord 8(6): 471-476.

36. Franz MJ, VanWormer JJ, Crain AL, Boucher JL, Histon T, et al. (2007) Weight-loss outcomes: a systematic review and meta-analysis of weight-loss clinical trials with a minimum 1-year follow-up. J Am Diet Assoc 107(10): 1755-1767.

37. Curioni CC, Lourenco PM (2005) Long-term weight loss after diet and exercise: a systematic review. Int J Obes (Lond) 29(10): 1168-1174.

38. Azziz R, Dumesic DA, Goodarzi MO (2011) Polycystic ovary syndrome: an ancient disorder? Fertil Steril 95(5):1544-1548. 
(CC) This work is licensed under Creative (CC) Commons Attribution 4.0 License BY DOI: 10.19080/JGWH.2021.21.556069

\section{Your next submission with Juniper Publishers} will reach you the below assets

- Quality Editorial service

- Swift Peer Review

- Reprints availability

- E-prints Service

- Manuscript Podcast for convenient understanding

- Global attainment for your research

- Manuscript accessibility in different formats

( Pdf, E-pub, Full Tsext, Audio)

- Unceasing customer service

Track the below URL for one-step submission https://juniperpublishers.com/online-submission.php 